\title{
Editorial
}

\section{Endothelin in heart failure: a promising therapeutic target?}

\section{Why we need more treatments for heart failure}

Angiotensin converting enzyme (ACE) inhibitors have revolutionised the treatment of heart failure caused by left ventricular systolic dysfunction. ${ }^{1}$ However, few patients with heart failure are rendered asymptomatic by treatment with an ACE inhibitor or are able to lead a normal, active life. Their prognosis also remains dismal. In the treatment arm of the Studies of Left Ventricular Dysfunction (SOLVD), $35 \%$ of the relatively young patients (average age 61 years) with so-called mildly to moderately severe heart failure who were randomised to receive enalapril died within three and a half years of follow up. Forty eight per cent died or were admitted to hospital for worsening heart failure, while $69 \%$ were admitted to hospital at least once during the trial. ${ }^{1}$

The initial success of ACE inhibitors has been followed by many false dawns in heart failure therapeutics. With the possible exception of $\beta$ blockers, no new treatment for cardiac failure has succeeded in the 20 years since captopril was developed. Pathophysiological studies and experience with drugs such as hydralazine, nitrates, flosequinan, phosphodiesterase inhibitors, prostacyclin, and ibopamine have strongly suggested that the most effective therapeutic strategy in heart failure is arterial and venous vasodilatation via inhibition of endogenous vasoconstrictor neuroendocrine pathways. ${ }^{1}$ These actions, exemplified by ACE inhibitors, lead to improved skeletal muscle and vital organ perfusion. Such a strategy should not result in activation of other neuroendocrine pathways or direct inotropic stimulation of the heart, though ventricular function can improve in response to a fall in cardiac preload and afterload. Furthermore, ACE inhibitors probably protect the heart from harmful neuroendocrine activation in ways that we do not fully understand. Prevention of further ischaemic events in patients who have underlying ischaemic heart disease (that is, most patients with heart failure) is a hitherto underappreciated and probably important mechanism by which drugs might improve prognosis in heart failure. Interestingly, the only other encouraging prospect in the field of heart failure therapeutics at present is the adrenoblocker carvedilol, a drug which antagonises the action of the sympathetic nervous system by blocking post-synaptic $\alpha_{1}, \beta_{1}$, and $\beta_{2}$ adrenoceptors. ${ }^{2}$

\section{Synthesis and actions of the endothelins}

The endothelins are a family of three structurally and functionally similar peptides, each of 21 amino acid residues. ${ }^{34}$ The processing pathway for endothelin-1, the major endothelin isoform synthesised in human blood vessels, is reminiscent of angiotensin II. Initially, a large prepropolypeptide, preproendothelin-1, consisting of about 200 amino acid residues, is synthesised. A biologically inactive intermediate propeptide, big endothelin-1, is subsequently formed through proteolytic cleavage. Finally, a metalloprotease, endothelin converting enzyme (ECE) generates the biologically active mature peptide endothelin-1 consisting of 21 amino acid residues.

The vasoconstrictor action of endothelin-1 is mediated through two high affinity endothelin receptor subtypes, ETA and ETB, on vascular smooth muscle cells. ${ }^{4}$ ETB receptors are also present on the vascular endothelium where they mediate vasodilatation, probably by release of nitric oxide and prostanoids. Despite the presence of these endothelial ETB receptors, the characteristic biological effect of exogenously administered endothelin-1 is slow onset and sustained vasoconstriction. ${ }^{45}$ Indeed, endothelin-1 is the most potent vasoconstrictor known, demonstrating ten times greater vasoconstrictor potency in vitro than angiotensin II. ${ }^{3}$ Endothelin- 1 may also augment the vasoconstrictor effects of arginine vasopressin, the renin angiotensin aldosterone system, and the sympathetic nervous system. Endothelin-1 has particularly pronounced vasoconstrictor effects in the renal vasculature and reduces renal plasma flow, glomerular filtration rate, and sodium excretion-actions that are analogous to those of angiotensin II. Again like angiotensin II, endothelin-1 has a direct positive inotropic action on the heart, although experimentally the effect of exogenous endothelin-1 in vivo depends upon the extent of simultaneous coronary vasoconstriction that is produced. Endothelin-1 also has potent effects on cell growth, inducing protooncogene expression and increasing DNA synthesis in vascular smooth muscle cells and cardiomyocytes. ${ }^{4}$

Because endothelin-1 has many similar biological properties to angiotensin II it too may play an important pathophysiological role in the various cardiac, vascular, and renal abnormalities typical of heart failure.

\section{Endothelin-1 in heart failure}

Though secreted predominantly abluminally by vascular endothelial cells, plasma concentrations of endothelin-1 are increased two- to threefold in patients with heart failure irrespective of aetiology. ${ }^{6-8}$ As with other neurohormones, plasma concentrations of endothelin-1 are increased in proportion to the symptomatic and haemodynamic severity of heart failure. ${ }^{78}$ In distinction to other neurohormones, however, plasma endothelin-1 concentrations correlate particularly closely with pulmonary vascular resistance. ${ }^{8}$ Importantly, and as with angiotensin II and noradrenaline, plasma big endothelin-1 concentrations are of prognostic significance, predicting worsening heart failure, need for admission to hospital, and death. ${ }^{9}$ In one study, plasma big endothelin-1 concentration was a more powerful predictor of prognosis than left ventricular ejection fraction, plasma renin activity, plasma atrial natriuretic factor concentration, or systemic vascular resistance. ${ }^{9}$

From this brief outline, it seems that endothelin-1 could contribute in an analogous fashion to angiotensin II to the adverse pulmonary vascular, systemic vascular, 
and renal changes that characterise heart failure. The potent coronary vascular effects of endothelin-1 suggest that the peptide might also play a part in the genesis and pathophysiology of myocardial ischaemia and infarction. ${ }^{4}$

\section{Pharmacological inhibition of endogenous endothelin-1}

Studies in healthy volunteers have shown that administration through the brachial arterial of a locally active dose of the ECE inhibitor phosphoramidon (which prevents endothelial generation of mature endothelin-1 from big endothelin-1) or the selective ETA receptor antagonist BQ-123 causes substantial forearm vasodilatation. ${ }^{5}$ Systemic administration of the non-selective ETA and ETB receptor antagonist TAK-044 causes rapid onset, sustained, dose-dependent peripheral vasodilatation and hypotension in young healthy male volunteers. ${ }^{10}$ Heart rate, stroke index, and cardiac index all increased after administration of TAK-044. These recent experiments indicate that endogenously generated endothelin-1 plays an important physiological role in regulating vascular resistance and blood pressure in humans.

In patients with heart failure, local brachial arterial ECE inhibition and ETA receptor blockade both cause significant forearm vasodilatation. Importantly, these effects are seen in patients concurrently treated with diuretics, digoxin, and large doses of ACE inhibitor. ${ }^{11}$ Systemic endothelin receptor blockade has also been studied in patients with heart failure, but only after ACE inhibitor withdrawal. ${ }^{12}$ Systemic administration of the non-selective ETA and ETB receptor antagonist bosentan reduced pulmonary artery wedge pressure, systemic vascular resistance, and mean arterial pressure. Cardiac index was increased without causing reflex tachycardia. Pulmonary vascular resistance was decreased more than systemic vascular resistance, an effect that contrasts with most other vasodilators studied in heart failure. Animal and patient studies have also suggested an apparent differential alteration in endothelin receptor sensitivity in heart failure whereby there is a diminished response to ETA receptor stimulation and a relatively greater vasoconstrictor response to ETB receptor stimulation. ${ }^{11}$

It is likely that the endothelin system interacts importantly with the other neuroendocrine pathways which are activated in heart failure, in particular the renin angiotensin aldosterone and sympathetic nervous systems. Angiotensin II increases endothelin-1 secretion from cultured endothelial cells, and a correlation between plasma angiotensin II and endothelin-1 concentration has been demonstrated in patients with heart failure. ${ }^{313}$ Captopril inhibits endothelial production of endothelin-1 in vitro, and high dose lisinopril has been reported to suppress plasma endothelin-1 concentrations in vivo in patients with heart failure. ${ }^{14}{ }^{15} \mathrm{~A}$ fall in plasma endothelin-1 was recently reported to predict a favourable functional and haemodynamic response to treatment with carvedilol in patients with heart failure. ${ }^{16}$ This may be because carvedilol suppresses the renin angiotensin system or because the sympathetic nervous system directly influences endothelin-1 synthesis and secretion. It is interesting to speculate that endothelin-1 inhibition might contribute to the beneficial actions of both ACE inhibitors and carvedilol in heart failure.

\section{Summary}

The results of early acute haemodynamic studies with anti-endothelin agents are promising. Much still needs to be done, however, before endothelin antagonism is established as a therapeutic strategy in heart failure. We need to know, for example, whether the haemodynamic effects of anti-endothelin drugs are sustained. We need to ensure that there is no reflex activation of other neuroendocrine systems and, preferably, to demonstrate neuroendocrine suppression. Characterisation of the renal actions of endothelin receptor antagonists will also be important.

Perhaps the most pressing issue in the development of these agents is elucidation of the role of the endothelial ETB receptor in heart failure. It is now clearly shown that vascular smooth muscle ETB receptors can mediate vasoconstriction in human blood vessels and that these receptors may be particularly important in heart failure. ${ }^{11}$ The effect of selective ETB receptor blockade in humans in vivo is not currently known, however, and whether endothelial ETB receptors might tonically offset ETA and ETB receptor mediated smooth muscle contraction remains conjectural. This question is directly relevant to whether selective ETA or non-selective ETA and ETB receptor antagonism might be the better therapeutic strategy in heart failure. ECE inhibition may become another therapeutic option in due course, but at present no specific and selective inhibitors of the enzyme have been developed. The recent demonstration that the selective ETA receptor antagonist BQ-123 improves long term survival in rats with heart failure induced by myocardial infarction suggests that anti-endothelin strategies may hold great therapeutic promise in heart failre. ${ }^{17}$

MICHAEL P LOVE* JOHN JV MCMURRAY Medical Research Council Clinical Research Initiative in Heart Failure, Wolfson Building,

University of Glasgow,

Glasgow G12 8QQ

*MPL is supported by a Bristol-Myers Squibb Cardiovascular Fellowship

1 Dargie HJ, McMurray JJV. Diagnosis and management of heart failure. $B M \Im$ 1994;308:321-8.

2 Packer M, Bristow MR, Cohn JN, Colucci WS, Fowler MB, Gilbert EM, et al. The effect of carvedilol on morbidity and mortality in patients with chronic heart failure. N Engl $\mathcal{F}$ Med 1996;334:1349-55.

3 Yanagisawa M, Kurihara H, Kimura S, Tomobe Y, Kobayashi M, Mitsui Y, et al. A novel potent vasoconstrictor peptide produced by vascular endothelial cells. Nature 1988;332:411-5.

4 Haynes WG, Webb DJ. The endothelin family of peptides: local hormones with diverse roles in health and disease. Clin Sci 1993;84:485-500.

5 Haynes WG, Webb DJ. Contribution of endogenous generation of endothelin-1 to basal vascular tone. Lancet 1994;344:852-4.

6 McMurray JJ, Ray SG, Abdullah I, Dargie HD, Morton J. Plasma endothelin in chronic heart failure. Circulation 1992;85:1374-9.

7 Rodeheffer RJ, Lerman A, Heublein DM, Burnett JC Jr. Increased plasma concentrations of endothelin in chronic heart failure in humans. Mayo Clin Proc 1992;67:719-24.

8 Cody RJ, Haas GJ, Binkley PF, Capers Q, Kelley R. Plasma endothelin correlates with the extent of pulmonary hypertension in patients with correlates with the extent of pulmonary hypertension in
chronic congestive heart failure. Circulation 1992;85:504-9.

9 Pacher R, Stanek B, Hlsmann M, Koller-Strametz J, Berger R, Schuller M, et al. Prognostic impact of big endothelin-1 plasma concentrations compared with invasive hemodynamic evaluation in severe heart failure. $\mathcal{f} \mathrm{Am}$ parl Cardiol 1996;27:633-41.

10 Haynes WG, Ferro CJ, OKane KPJ, Somerville D, Lomax CC, Webb DJ. Systemic endothelin receptor blockade decreases peripheral vascular resistance and blood pressure in humans. Circulation 1996;93:1860-70.

11 Love MP, Haynes WG, Gray GA, Webb DJ, McMurray JV. Vasodilator effects of endothelin converting enzyme inhibition and endothelin ETA receptor blockade in chronic heart failure patients treated with ACE inhibitors. Circulation 1996;94:2131-7.

12 Kiowski W, Stsch G, Hunziker P, Muller P, Kim J, Oechslin E, et al. Evidence for endothelin-1 mediated vasoconstriction in severe chronic heart failure. Lancet 1995;346:732-6.

13 Good JM, Nihoyannopoulos P, Ghatel MA, Crossman D, Bloom SR, Clark $P$, et al. Elevated plasma endothelin concentrations in heart failure; an effect of angiotensin II? Eur Heart ₹ 1994;15:1634-40.

14 Momose N, Fukuo K, Morimoto S, Ogihara T. Captopril inhibits endothelin-1 secretion from endothelial cells through bradykinin. Hypertension 1993;21:921-4.

15 Davidson NC, Coutie WJ, Webb DJ, Struthers AD. Hormonal and renal differences between low dose and high dose angiotensin converting enzyme inhibition in patients with chronic heart failure. Heart enzyme inhibition

16 Krum H, Gu A, Wilshire-Clement M, Sackner-Bernstein J, Goldsmith R, Medina N, et al. Changes in plasma endothelin-1 levels reflect clinical response to $\beta$-blockade in chronic heart failure. Am Heart $\mathcal{f} 1996$; response to

17 Sakai S, Miyauchi T, Kobayashi M, Yamaguchi I, Goto K, Sugishita Y. Inhibition of myocardial endothelin pathway improves long term survival in heart failure. Nature 1996;384:353-5. 